\title{
Using Deep Learning to Detect Polyethylene Terephthalate (PET) Bottle Status for Recycling
}

By Hellen Wanini Mwangi \& Mpai Mokoena Abstract- Following the recent ban of plastic waste import by China, many developed countries face challenges with their huge amount of plastic waste. Some of the countries have diverted their waste to other developing East-Asia countries like Philippines, Vietnam and Malaysia. However, Malaysian government has taken strict action to send back over 3000 tons of plastic waste to its origin citing contamination. The aim of this paper is to establish mechanisms to detect the status of post-consumer PET bottles for recycling. By encouraging recycling of clean pet bottles, we ensure high quality bottles for recycling. A research based as well as experimental design approach was adopted to develop mechanisms to detect PET bottle status. During the experiment, various PET bottles were collected and images captured. A total of 1749 images were captured using raspberry Pi camera.

Keywords: recyclable pet bottles, squeeze net, recycling, predictions, deep learning, incentives.

\section{GJCST-G Classification: 1.2 .6}

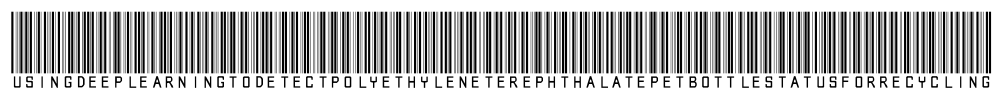

Strictly as per the compliance and regulations of:

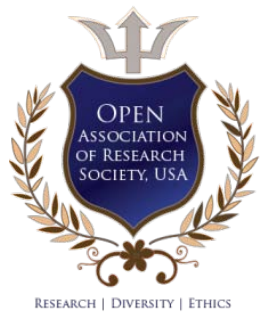

(c) 2019. Hellen Wanini Mwangi \& Mpai Mokoena. This is a research/review paper, distributed under the terms of the Creative Commons Attribution-Noncommercial 3.0 Unported License http://creativecommons.org/licenses/by-nc/3.0/), permitting all non commercial use, distribution, and reproduction in any medium, provided the original work is properly cited. 


\title{
Using Deep Learning to Detect Polyethylene Terephthalate (PET) Bottle Status for Recycling
}

\author{
Hellen Wanini Mwangi ${ }^{\alpha} \&$ Mpai Mokoena ${ }^{\sigma}$
}

Abstract-Following the recent ban of plastic waste import by China, many developed countries face challenges with their huge amount of plastic waste. Some of the countries have diverted their waste to other developing East-Asia countries like Philippines, Vietnam and Malaysia. However, Malaysian government has taken strict action to send back over 3000 tons of plastic waste to its origin citing contamination. The aim of this paper is to establish mechanisms to detect the status of post-consumer PET bottles for recycling. By encouraging recycling of clean pet bottles, we ensure high quality bottles for recycling. A research based as well as experimental design approach was adopted to develop mechanisms to detect PET bottle status. During the experiment, various PET bottles were collected and images captured. A total of 1749 images were captured using raspberry $\mathrm{Pi}$ camera. The images belong to 4 different classes; seal, cap, seal \& cap, no seal, cap \& content. Deep Learning technology (Squeeze Net) was used to train the PET bottle images. After training the bottle images, the model achieved $98 \%$ accuracy with correct bottle status recognized. The trained model is then deployed on a raspberry pi to detect PET bottles in real time when using a reverse vending machine. When measuring prediction performance, the model showed a delay of 0.018 to 0.022 seconds per prediction using Intel CPU. Whereas measuring the prediction performance in raspberry pi which is 5 to 10 times slower than the Intel CPU with a delay of 0.1 to 0.25 seconds per prediction.

Keywords: recyclable pet bottles, squeeze net, recycling, predictions, deep learning, incentives.

\section{INTRODUCTION}

$\longrightarrow$ urrently, recyclers are looking for more from shredders. According to Plastic Recycling Technology 2019, new ways to boost productivity, quality and profitability are being sought. There is more demand for quality Polyethylene Terephthalate (PET) bottles. "Purity rates of $98 \%$ are now being sought as standard, which means there's no longer a place for contaminated plastics PET bottles. Furthermore over a million plastic bottles are bought every minute globally, by 2021 this number is expected to increase by $20 \%$. This means firms have to prioritize quality PET bottles through reduce, reuse, recycle principles." Consequently, when the three principles of recycling are not followed, over 8 million tonnes of plastic bottles enter the sea every year (Plastic Oceans, 2017). Thus, efforts to collect the plastic waste have been expressed

Author av: e-mails: hwaninitech@gmail.com, mandymkn4@gmail.com by many developing and developed countries to recycle the plastic PET bottle waste.

According to The Guardian 2017, annual production of plastic bottles is expected to increase from 500 billion to half a trillion by 2021 (figure1).

\section{Humans produce almost 20,000 plastic bottles every second}

Global PET plastic bottle production

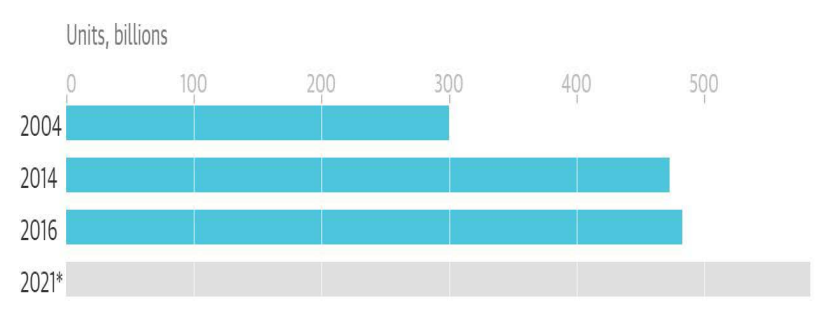

Figure 1: PET bottle production

\section{a) Problem statement}

PET is the most widely produced, consumed and recycled plastic in the world. Although PET is recyclable, a huge quantity is discarded. Consequently, the huge amount of PET that is not recycled poses a threat to the environment, negatively affecting marine life and human health. As a result, this paper proposes a solution that can ensure the quality PET bottles are collected and recycled.

b) Objectives

Based on the topic, the paper is guided by several research objectives:

- To establish effective mechanisms for postconsumer PET bottles status recognition in real-time using reverse vending machines.

- Build prediction system to recognize PET bottle status using sensors and deep learning.

- $\quad$ To test and evaluate the performance of the deep learning model in bottle status recognition

c) Methodology

A qualitative and quantitative method as well as experimental method was used. The reason for selecting these methods. Both qualitative and quantitative methods were used to analyze the problem. Experiment was conducted by collecting post-consumer PET bottles to train the model. The experiment helped to 
verify the solution which is the identification of pet bottle status using deep learning.

This paper is structured as follows: Section 2 covers recycling frameworks; Section 3 compares existing solutions; Section 4 discusses the proposed solution; Section 5 is about the prototype development; Lastly, in Section 6 the results are discussed.

\section{Recycling FrameWORKS}

\section{a) SDGs}

The term SDGs refer to the Sustainable Development Goals set by the United Nations Development Program (UNDP).Several of these touches on waste management, health and marine life.

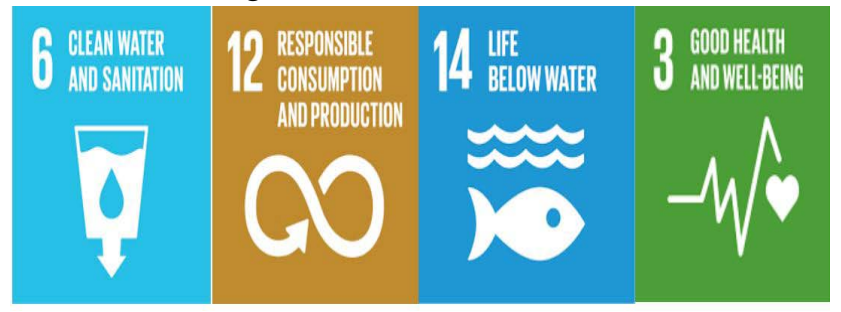

Source: UNDP

Figure 2: Sustainable Development Goals this paper touches on

By collecting post-consumer PET bottles for recycling, we ensure a clean environment, marine life as well as good health from a clean environment. It also ensures that there is responsible production and consumption of PET bottles produced and end-of-life. The principle of Extended Producer Responsibility guides manufacturers to ensure responsible production.

\section{ili. Existing Solutions}

\section{a) Overview}

In this section, the paper compares several case studies in relation to plastic PET bottle waste management in different countries. The cases are based on: 1) Successful models of reverse vending machines for post-consumer PET bottle collection, case of Sweden; 2) The PETCO model by Plastic Recycling Company of South Africa; 3) The case of Japan.

\section{b) Returpack Sweden}

The Returpack case study on Deposit Refund Systems is insightful. The system takes care of four types of beverage containers PET bottles being one of them. The collection systems promote the collection of recyclables and waste with the provision of economic incentives. There are established deposit refund systems in Sweden and reward points collection systems in place. To have a clear understanding of how the deposit refund systems work in Sweden, a research on Sweden Deposit Refund System has been done by way of interview and obtained first-hand information from a resident citizen of Sweden.
According to the interview results, the consumers return the bottles to the drop-off points where the vending machines are located. However, the system does not take into consideration the quality of bottles returned such as the removal of cap, label or content or cleanliness. Upon returning the bottles, consumer gets a coupon which can be redeemed at the stores. The vending machine scans the barcode of the bottle and it rejects any bottle that is not Swedish. As a result, the system encourage recycling of PET bottles by providing rewards.

From the research findings, Sweden depositrefund system is mandatory, it began in the 1970s in order to achieve the $75 \%$ recycling rate indicated by the government. The key stakeholders of the system include the can manufacturer, breweries and retailers who established a company by the name Returpack to set up collection and recycling system for aluminium cans. Also, Returpack-PET (AB Svenska Returpack PET) was established for PET bottles in 1994. (Tojo, 2011). Prior to Sweden having its own PET recycling plant, the PET bottles were sent to Germany for cleaning and recycling. In an effort to reduce PET bottle waste, a recycling company was established in 2006-2007. As a result, Sweden achieved a recycling rate of $90 \%$ for pet bottles (The Locals.Se, 2018)

\section{c) PETCO South Africa}

PETCO is a South African company which was incorporated in 2004 to self-regulate recycling of postconsumer PET bottles. PETCO is guided by the principle of Extended Producer Responsibility. It Involves various stakeholders in its business model which makes it a success. Stakeholders involved are raw material producers, converters, brand owners', retailers, consumers, recyclers and donors. Extended producer responsibility ensures the end of life of the products which the companies produce.

The country's recycling rate exceeded expectations in increasing from 52\% in 2015 to $55 \%$ in 2016(Post \& Number, 2018). In 2017, 2 billion PET bottles were recycled, the recycling rate was $65 \%$. PETCO South Africa has embraced a circular economy paying much attention to achieving the Sustainable Development Goals (SD Goals: 14, 12, 6, and 3) as shown in figure 2.

Another contribution to PETCO South Africa success in recycling is the existence of state-of-the-art recycling facilities. Also, recyclers can buy PET bottles from collectors with the fee collected from the voluntary fee paid by importers and manufacturers of PET bottle products.

In a 2017 report by Plastics SA, the major challenge faced in the recycling of plastic bottle waste is getting access to good quality, clean bottles before they reach the landfills. 


\section{d) Plastic Recycling in Japan}

Waste management in Japan is successful due to waste separation practice. For example, the first house rules for a tenant from a landlord is about waste separation. Kamikatsu in Japan has a collection centre for recyclables. The consumers return bottles and are awarded points. After the points accumulate, the residents can enjoy discounts for basic services such as housing, water, health etc.

\section{Proposed Solution}

In this paper a reverse vending machine with capability to identify bottle status real-time is proposed. This is the contribution to already existing solutions in collecting post-consumer PET bottles. The purpose of the solution is to encourage the return of recyclable quality PET bottle which

\section{a) How it works}

When the consumers finish with the pet bottles, they clean and return the empty bottles to a collection centre/drop-off point, there the bottle is put in the reverse vending machine, the bottle status is identified based on the following features; With seal or without seal, with cap or without cap, with cap and seal, or if it has no cap, content and seal. Awarding of incentives depends on the status and for each status, the amount awarded varies.

Figure 3 shows bottle status recognition which varies from low incentives for not clean bottles to high incentives for clean ones.

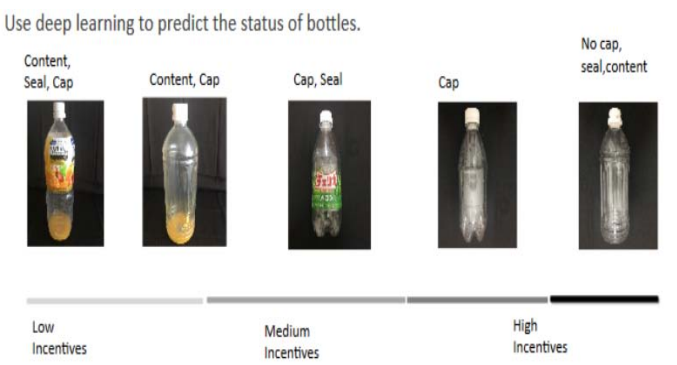

Figure 3: Bottle Status Recognition

Using deep learning technology to detect the status of the PET bottle in real-time in a reverse vending machine and give different amount of incentive as shown on the figure above.

\section{b) Comparison and Originality of the proposed solution to existing solutions}

Table 1 shows a comparison of the proposed solution to existing solutions. The originality of the proposed solution is 1) it adopts divergent incentives (the amount of incentives is decided based on the bottle status), and 2) the bottle status is recognized automatically.

\section{Table 1: Originality}

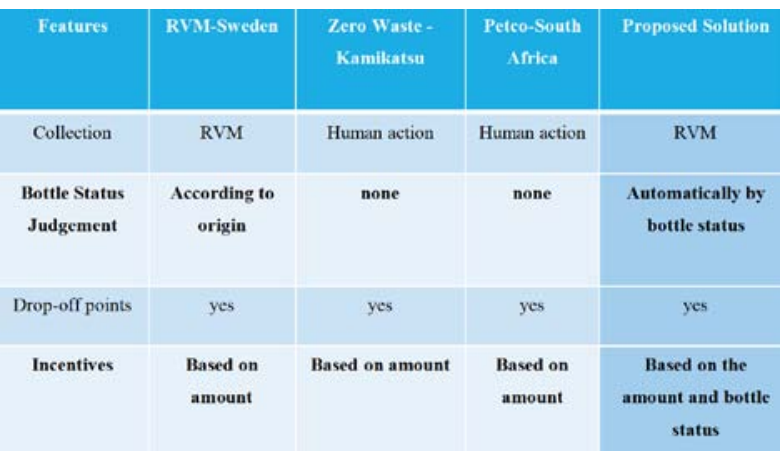

\section{Solution Development}

\section{a) System configuration}

In the reverse vending machine, we have a range/object sensor, a camera sensor and controller raspberry pi. When the bottle is put in the machine, the range sensor detects the bottle and triggers the camera to take photos. Figure 4 shows an overview of reverse vending machine with PET bottle status recognition.

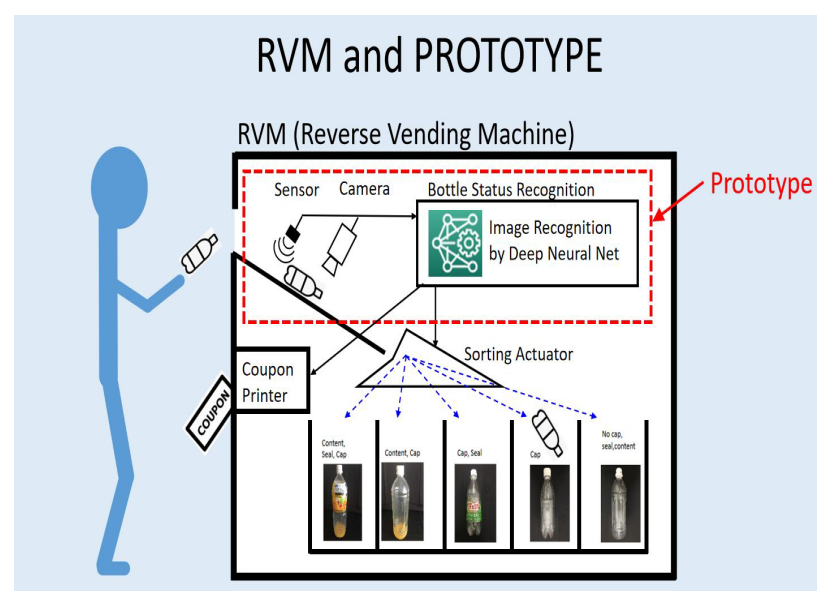

\section{Figure 4: Reverse Vending Machine}

In the taking photo process of training images, the images are saved in a network server (Samba) directory created on Linux computer.

During the prediction process, the bottle put in the reverse vending machine is captured and its image is saved on SD card in the raspberry pi. Using deep learning model (Squeeze Net) deployed on the raspberry pi, the bottle image is predicted and results displayed on the dashboard as shown in figure 5 below. 

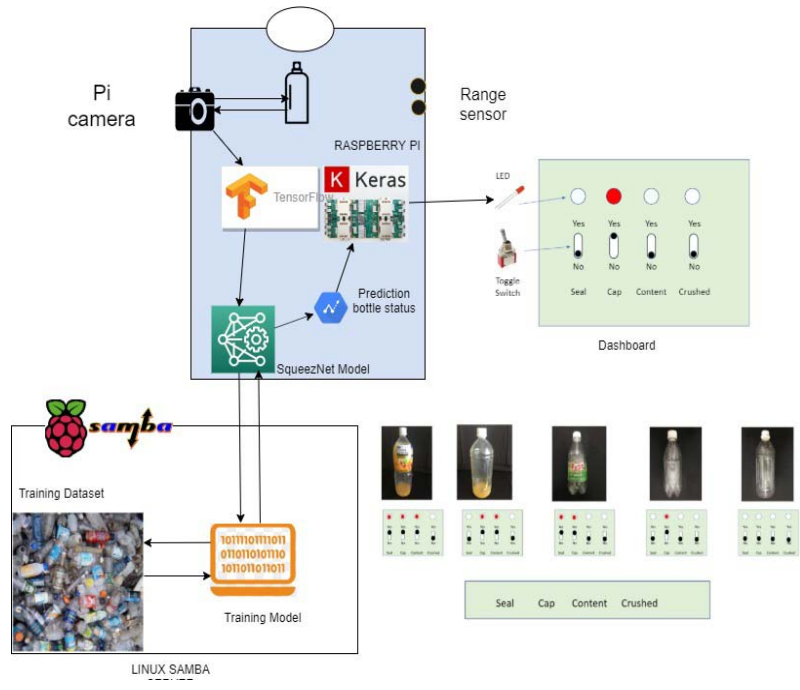

Figure 5: System Configuration Diagram

\section{b) Hardware configuration}

The configuration involves several devices. There are requirements in order to configure all the devices to work well. Below is a list of the requirements:

1. Range sensor (HC-SR04)

2. Raspberry pi camera

3. The raspberry pi 2 model $B$

4. PCB (A circuit board)

5. Wires

6. Four LEDs

7. Four toggle switches corresponding to each LED

Four toggle switches are connected to four GPIOs of Raspberry pi, so that the software on Raspberry pi can sense the status of the switches. This is used in the function of taking images for the training datasets. Four LEDs are connected to other four GPIOs of Raspberry pi, so that the software on Raspberry pi can turn on or off individually. This is used in the function of taking images for prediction to display the prediction result.

\section{c) Generating Training dataset}

The training dataset requires a label which corresponds to the status of the bottle for each image of the bottle, because the supervised machine training is used. In the prototype, two toggle switches out of four are used to indicate the label of the image. The status of bottles which must be recognized are the existence of seal, cap. So the number of bottle status is four: a) only seal exists, b) both seal and cap exist, c) only cap exists, and d) no seal and no cap exists. The four statuses are coded as binary values as a) 1000 , b) 1100, c) 0100, d) 0000 . Each binary digit is assigned to each toggle switch, so the user of the prototype easily specify the state of the bottle by setting the toggle switches. This feature is useful for speeding up the training dataset generation process.
Figure 6 shows the file directory structure of the image data for deep learning training. The directories "1000", "1100", "0100" and "0000" correspond to the bottle status a) to d). The captured image data is automatically saved to the corresponding directory. The images are saved in respective directories according to their classes. For example images of seal, cap, cap and seal are saved in 1000,0100, and 1100 respectively.

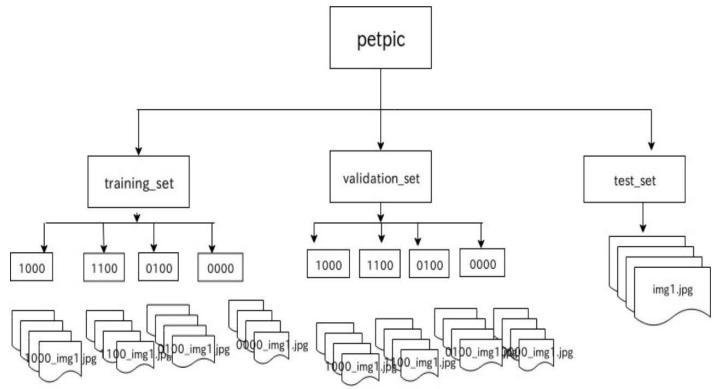

Figure 6: Dataset organizational structure

\section{Results and Discussions}

\section{a) Performance of Deep Neural Network}

Squeeze Net (landola, Forrest N., et al, 2016) is selected for the neural network because it is small enough to be executed on Raspberry pi. Using the Squeeze Net model successfully trained the dataset. Total parameters are 541,332. Training parameters are 540,812 and non-trainable parameters are 540 . Some of the hyper parameters are learning rate, weight decay (L2), 20 epochs and the 'accuracy' metrics to show the results. Figure 7 shows the loss and accuracy during the training going on
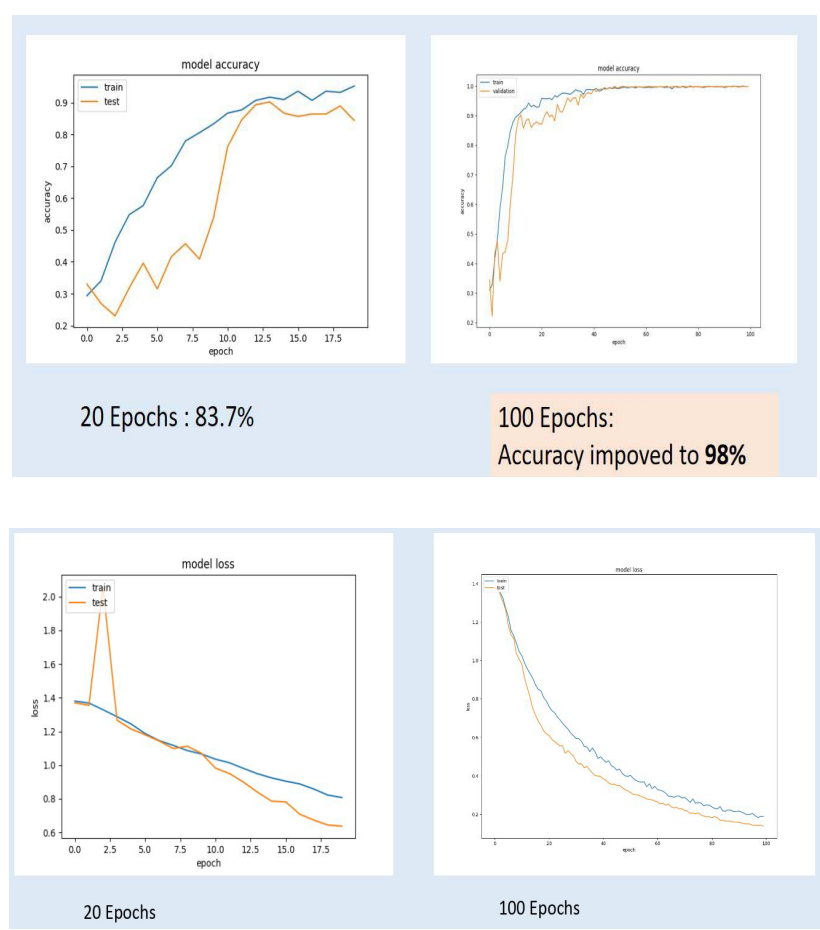

Figure 7: Visualizing training process 
Loss

Model loss is a value that tells us how well or bad our model classifier performed at each iteration. The lower the loss value the better the model performed and vice versa. It is easy for people to predict images with their human eyes but in deep learning it is difficult due to various challenges. Therefore, the need to apply a loss function in our model. There is no universal loss function that works for all classifications.

\section{Accuracy}

Accuracy of a model is determined after learning has taken place. Then the test samples are fed to our model to determine the accuracy. of predictions

Accuracy $=$ No of correct predictions $/$ Total no

The accuracy in the training of pet bottles is $83.7 \%$ for 20 epochs .After increasing the number of epochs, training accuracy improved. The results are $98 \%$ accuracy.

\section{b) Measuring the prediction performance}

The prediction results shown in table 2 shows the prediction performance on Intel CPU which were 0.022 seconds in the 1 st test and 0.018 seconds in the 2nd test. Since the raspberry pi CPU is 5 to 10 times slower than the Intel CPU, the approximated delay time is 0.1 seconds to 0.25 seconds per prediction.

Table 2: Squeeze Net performance results in predicting the images

\begin{tabular}{|l|l|l|}
\hline Environment & $\begin{array}{l}\text { General Performance } \\
\text { (In terms of prediction } \\
\text { time) }\end{array}$ & $\begin{array}{l}\text { Actual delay using } \\
\text { SqueezeNet (per prediction) }\end{array}$ \\
\hline PC with GPUs & $\begin{array}{l}80^{*} \text { faster than Intel } \\
\text { core i3 }\end{array}$ & - \\
\hline PC with CPU & $\begin{array}{l}0.66 \text { seconds using } \\
\text { Intel core i3 }\end{array}$ & $\begin{array}{l}1^{\text {n }} \text { test } 0.022 \text { seconds } \\
2^{\text {nd }} \text { test } 0.018 \text { seconds }\end{array}$ \\
\hline $\begin{array}{l}\text { Raspberry pi } \\
\text { CPU only }\end{array}$ & $\begin{array}{l}5-10 * \text { slower than } \\
\text { Intel core i3 }\end{array}$ & $\begin{array}{l}\text { Approximately } 0.1 \sim 0.25 \\
\text { seconds }\end{array}$ \\
\hline
\end{tabular}

\section{ReFerences Références Referencias}

1. Bengio, Y., 2009. Learning deep architectures for Al. Foundations and trends ${ }^{\circledR}$ in Machine Learning, 2(1), pp.1-127.

2. Camera Exposure matrix. Retrieved from:https://publiclab.org/notes/MaggPi/07-022018/camera-exposure-matrix

3. Chollet, F., 2015. Keras: Deep learning library for theano and tensor flow. URL: https://keras. io/k, 7(8), p.T1.

4. Gay, W., 2018. Pi Camera. In Advanced Raspberry Pi (pp. 493-499). Apress, Berkeley, CA.

5. Gopalakrishnan, K., Khaitan, S. K., Choudhary, A. and Agrawal, A., 2017. Deep Convolutional Neural
Networks with transfer learning for computer visionbased data-driven pavement distress detection. Construction and Building Materials, 157, pp.322330.

6. Iandola, F., \& Keutzer, K., 2017, October. Small neural nets are beautiful: enabling embedded systems with small deep-neural-network architectures. In Proceedings of the Twelfth IEEE/ACM/IFIP International Conference on Hardware/Software Codesign and System Synthesis Companion (p. 1). ACM.

7. landola, F. N., Han, S., Moskewicz, M. W., Ashraf, K., Dally, W. J. and Keutzer, K., 2016. Squeeze Net: Alex Net-level accuracy with 50x fewer parameters and $<0.5 \mathrm{MB}$ model size. Ar Xiv preprint arXiv:1602.07360.

8. Jambeck, J. R., Geyer, R., Wilcox, C., Siegler, T. R., Perryman, M., Andrady, A., Narayan, R. and Law, K.L., 2015. Plastic waste inputs from land into the ocean. Science, 347(6223), pp.768-771.

9. LeCun, Y., Bengio, Y. and Hinton, G., 2015. Deep learning. nature, 521(7553), p.436.

10. $\mathrm{Pi}, \mathrm{W}$., 2017. GPIO Interface library for the Raspberry Pi. URL: http://wiringpi. com/(visited on 2017-07-13).

11. Rawat, W. and Wang, Z., 2017. Deep convolutional neural networks for image classification: A comprehensive review Neural computation.

12. Rawat, W. and Wang, Z., 2017. Deep convolutional neural networks for image classification: A comprehensive review. Neural computation, 29(9), pp.2352-2449.

13. Setting up a samba server as a stand-alone server. Retrievedfrom:https://wiki.samba.org/index.php/Sett ing_up_Samba_as_a_Standalone_Server

14. https://www.theguardian.com/environment/2017/jun /28/a-million-a-minute-worlds-plastic-bottle-bingeas-dangerous-as-climate-change

15. Voulodimos, A., Doulamis, N., Doulamis, A. and Protopapadakis, E., 2018. Deep learning for computer vision: A brief review. Computational intelligence and neuroscience, 2018.

16. Arun David Ambrose., 2017. PET recycling - a means to save the planet. https://www.beroeinc.com/whitepaper/pet-recycle/

17. The Locals se., 2019. That's pant! The story behind Sweden's bottle recycling scheme. Retrieved from: https://www.thelocal.se/20180328/thats-pant-thestory-behind-swedens-bottle-recycling-system 